\title{
Analytical, numerical and experimental study of the plastic buckling behavior of thick cylindrical tubes under axial compression
}

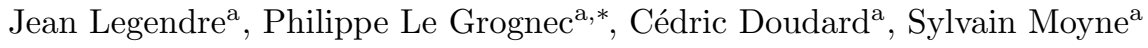 \\ ${ }^{a}$ ENSTA Bretagne, UMR CNRS 6027, IRDL, F-29200 Brest, France
}

\begin{abstract}
This paper deals with the elastoplastic buckling problem of thick cylindrical shells under axial compression. The main idea is to propose an analytical solution procedure which gives rise to reliable results in a straightforward and low time-consuming manner, for dimensioning purposes. Thick shells are thus considered as they are known to be less imperfection-sensitive than thin ones. First, experimental compression tests are performed thanks to a specific experimental set-up, which is designed so as to prevent again, as much as possible, from the imperfections due to boundary conditions. Then, numerical finite element computations are carried out, and it is shown that the choice of free edge boundary conditions (for which pre-critical deformations are supposed to be homogeneous) in the numerical model leads to the same critical load as obtained during the experimental tests. Next, the problem is solved analytically. The classical plastic buckling load, usually derived considering an homogeneous pre-critical state, is likely to agree with the numerical predictions in this particular case of free conditions, contrary to the cases of simply-supported or clamped edges actually displaying an heterogeneous pre-critical state. In return, the substantial thickness of the tubes considered experimentally makes it necessary to improve the well-known solution which appears to be in very bad agreement with the numerical/experimental critical values. On one hand, the issue of kinematics is discussed and, on the other hand, geometric non-linearities are taken into account in the model (pre-critical strains are no longer infinitesimal). Finally, the new analytical procedure derived in this paper shows a very good agreement with both numerical and experimental results.
\end{abstract}

Keywords: Plastic buckling, Cylindrical tubes, Axial compression, Geometric non-linearities

\section{Introduction}

The collapse of shell structures, especially when submitted to compressive loadings, is mainly due to buckling phenomena. Therefore, the mechanical design of such structures must include an analysis of the buckling behavior, implying the calculation of the critical loads and bifurcation modes. Thin shells usually

\footnotetext{
*Corresponding author.

Email address: philippe.le_grognec@ensta-bretagne.fr (Philippe Le Grognec)
} 
buckle in elasticity. In this context, bifurcation and stability of the shell are intrinsically related (see Koiter's theory), and a multitude of configurations (shell geometries and loading cases) have already been handled in the literature, both in an experimental and theoretical way.

The buckling problem is much more difficult to analyze when considering thicker structures, for which plasticity may occur before buckling. Hill [1] examined the uniqueness and stability criteria of a 3D elastoplastic continuum (by using the concept of "comparison elastic solid"), and highlighted the difference between bifurcation and stability in plasticity. The more significant feature is the existence of continua of bifurcation points in plastic buckling problems (instead of discrete ones in elasticity), which was discovered first by Cimetière [2] in the case of compressed rectangular plates. However, for obvious reasons, most of the theoretical results available in the literature at this time concern the plastic bifurcation at the tangent modulus critical value which corresponds to the minimum possible buckling load at which the structure may buckle in practice. Shanley [3] was the first to derive the tangent modulus critical load for a discrete model. Later, a few results have been obtained concerning beams or shells under various loadings and boundary conditions. In all cases, contrary to elasticity, the post-buckling analysis revealed an unstable behavior, namely the bifurcation load was found to be equal or very close to the maximum admissible load observed during the post-critical response.

The case of a circular cylindrical shell under axial compression is certainly one of the most considered cases in elastic/plastic buckling analyses. It corresponds both to one of the most critical buckling problem and to a realistic configuration usually encountered in practical applications (for instance, fuel tanks of space launch vehicles are typically cylindrical stiffened shells submitted to axial loads [4]). This fundamental problem is highly dependent on many parameters related to the geometry, the constitutive law and the boundary conditions, among others. That being said, except for very long cylinders whose buckling behavior may come closer to the one of a beam-like structure, roughly speaking, only two buckling mode types are prone to arise: usually a non-axisymmetric shape in the case of thin shells and an axisymmetric one for sufficiently thick shells, when plastic buckling is concerned.

In the past forty years, the problem of a cylindrical shell under axial compression was the subject of many experimental and numerical investigations (see [5, 6] for the pioneering works) and large differences have always been observed between numerical predictions and experimental results (the experimental critical loads sometimes falling down to less than half of the numerical values). These discrepancies are due to unavoidable imperfections in experiments: mainly geometric imperfections, but also imperfections related to loadings and boundary conditions, among others. This imperfection sensitivity was explained by many authors, such as Hutchinson [7] in his asymptotic analysis of plastic post-buckling. It is closely related to the unstable buckling response of such a problem, all the more so in plasticity. As far as plastic buckling is concerned, different critical values may be obtained, depending on the retained plasticity theory. In the case of axially compressed cylinders, Gellin $[8]$ analyzed the effects of an axisymmetric modal imperfection on the 
plastic buckling using the deformation theory, and comparisons between the predictions of both flow and deformation theories and experimental results were performed more recently in [9, [10] and [11. Ore and Durban [12] also derived semi-analytical values for the critical load of a cylinder under axial compression and various boundary conditions, with a special emphasis on axisymmetric modes. They showed again the discrepancy between the results provided by the flow and deformation theories of plasticity. In all cases, it turns out that the flow theory largely overpredicts the critical values, whereas the deformation theory gives rise to predictions in much better agreement with the experimental values, although the latter does not include the elastic unloading possibility (this major issue is known as the plastic buckling paradox). Tvergaard [13, 14] also analyzed the buckling behavior of cylindrical shells under axial compression, using the phenomenological theory of Christoffersen and Hutchinson [15] and assuming an initial axisymmetric sinusoidal (modal) shape. In the case of intermediate thicknesses, he obtained a bifurcation point leading to a non-axisymmetric mode just before the maximum load. Conversely, for very thick shells, the maximum load is found to coincide with the localization of the axisymmetric deformed shape at the ends (forming outward axial half-waves), in such a way that very thick shells are proved to collapse axisymmetrically.

Most of the analytical investigations on the plastic buckling of axially compressed cylindrical shells concern thus the axisymmetric case. In this context, one can mention Batterman [16] who derived first the following critical stress using the flow theory of plasticity and considering simply-supported end conditions:

$$
\sigma_{c}^{p}=\frac{E t}{R} \sqrt{\frac{4 H}{3\left[(5-4 \nu) E+4 H\left(1-\nu^{2}\right)\right]}}
$$

where $t$ denotes the shell thickness, $R$ the radius of the cylinder, and $E, \nu$ and $H$ represent the Young's modulus, the Poisson's ratio and the linear hardening modulus, respectively. The critical stress (1) may be also used in the case of built-in edges since almost the same sinusoidal mode shape is observed in both cases. In contrast, with free ends, the axisymmetric buckling mode looks like an exponential wave (the radial displacement being maximal at the ends of the cylinder and almost zero in the middle) and a critical stress equal to half of the preceding value is obtained (when considering a constant hardening modulus) [17]. This last result was derived in the particular case of a semi-infinite cylinder but Batterman showed that the critical stress barely depends on the (finite) length of the shell in practice (as soon as it is reasonably long).

Lastly, let us mention Mao and $\mathrm{Lu}$ [18] who considered the more general case of a cylindrical shell under combined axial and circumferential compressive loading stresses. In their study, the transverse shear is taken into account in order to analyze properly the interactive effect of stresses in both directions on the plastic buckling. Finally, Peek [19] derived analytical solutions for the immediate post-critical behavior of a cylinder under axial compression in the axisymmetric case only, but considering finite strains. 


\section{Outline of the paper}

The main objective of the present paper is to reconciliate both experimental and theoretical (numerical/analytical) points of view concerning the plastic buckling problem of a cylindrical shell under axial compression. The idea is to define a reference framework within which analytical, numerical and experimental critical loads coincide. This starting point will enable one to study the influence of the constitutive law and the initial conditions (such as residual stresses deriving from the manufacturing process) in both an experimental and a simple analytical way, in the context of marine structures.

In the literature, the deformation theory of plasticity or the corner theory are usually preferred as they predict more accurate critical loads than the flow theory (independently of any analysis of imperfection sensitivity). In this study, instead of comparing the merits of these different theories, our aim is to prove the ability of the $J_{2}$ flow theory of predicting plastic buckling loads. Despite the well-known significant influence of the imperfections on the buckling behavior of a cylinder under axial compression (Wang et al. 20. have lately experimented a decrease of more than $10 \%$ of the critical load even for quasi-perfect cylinders without intentionally made imperfections), we shall not account for any type of imperfection in this paper and will only analyze the behavior of a perfect cylinder. Very thick tubes will be considered so as to minimize the influence of geometric defects, as it is well-known that thicker tubes are less imperfection-sensitive. In return, boundary conditions have a strong effect on the plastic buckling of thick shells, compared to the case of elastic thin shells, in the fact that they give rise more or less to heterogeneous pre-critical deformations that strongly affect the critical values as initial imperfections, all the more so in plasticity. Stein 21] and Almroth 22] analyzed the buckling behavior of a cylindrical shell under axial compression, including in their formulation the effect of pre-buckling deformations due to the boundary conditions. Even if it is possible to derive explicit expressions for the pre-buckling deformed shape in simply-supported or clamped conditions (at least in elasticity), the buckling analysis can then only be performed using numerical approximations. Therefore, the idealized case of free edge conditions will be preferred here (as this configuration ensures an homogeneous pre-critical state and thus allows one to get rid of this type of imperfections).

First, Section 3 describes an original experimental set-up which has been developed with the objective of testing cylindrical tubes under axial compression with the edges remaining as free as possible. Experimental compression tests are carried out and the corresponding results are briefly discussed. Tensile tests are also conducted in order to calibrate the material law and numerical finite element computations are then performed, whose modeling hypotheses and results are presented thereafter. Experimental and numerical critical values are finally confronted and show a very good agreement.

This interesting result is very encouraging for the development of an analytical model where there is no need to take into account any kind of imperfection neither related to the geometry nor to the boundary conditions, in accordance with the previous numerical model. Section 4 summarizes first the main results 
of the plastic bifurcation theory, assuming the $J_{2}$ flow plasticity theory with the von Mises yield criterion and an isotropic hardening. Then, it is applied to the problem in hand. The classical closed-form expression first obtained for the critical stress rather differs from the previous experimental and numerical values, and two possible explanations are suggested and investigated. The choice of kinematics taking into account transverse shear effects is first discussed and, most importantly, the influence of geometric non-linearities is demonstrated and quantified. Finally, an analytical solution procedure is retained and allows one to recover the previous experimental critical value with a very good accuracy.

\section{Experimental and numerical analyses}

\subsection{Compression tests}

All the experimental tests have been achieved on a universal tensile/compression testing machine (MTS) with a $250 \mathrm{kN}$-force cell. The top end of the specimens is mounted on a fixed bracket whereas the bottom end rests on a movable bracket (see Figure 11. At the lower end, a ball joint ensures that the axial load is uniformly distributed on the bearing surface. Use is made of enforced displacements at a low strain rate (about $10^{-4} \mathrm{~s}^{-1}$ ) so as to fulfill quasi-static conditions. The tests are also post-processed with the help of a Digital Image Stereo-Correlation device (GOM Aramis).

While experimental compression tests on cylindrical shells are commonly performed with clamped edges in the literature for convenience purposes, a specific experimental set-up has been devised in this work, with the objective to reproduce as well as possible free edge boundary conditions. As seen in Figure 1, during compression, the cylindrical tubes are not radially constrained and both edges may move outwards freely thanks to the special care given to lubrication (friction is avoided as much as possible). In these conditions, no undesirable edge effects may arise and the pre-critical behavior appears to be quite homogeneous, as will be shown later.

Thick circular cylindrical tubes are tested with mean radius $R=11.5 \mathrm{~mm}$ and thickness $t=2 \mathrm{~mm}$ (see Figure 2). This particular low radius-to-thickness ratio $(R / t=5.75)$ ensures that plasticity will occur before buckling and prevents from any significant influence of geometric imperfections. Several samples of different lengths ( $L=50 \mathrm{~mm}$ and $L=75 \mathrm{~mm}$ ) are considered in order to check that the cylinder length is sufficiently high and has actually no influence on the buckling behavior in this particular range (conversely, an axisymmetric local buckling mode is actually obtained and not a global column-type buckling mode when considering such length-to-diameter ratios lower than 4, as recently shown in [23]). The material considered in this study is a 6060 aluminum alloy.

The specimens are compressed until an advanced post-buckling range and the typical deformed state (observed after unloading) is depicted in Figure 3 (three samples have been tested for each length, all of 


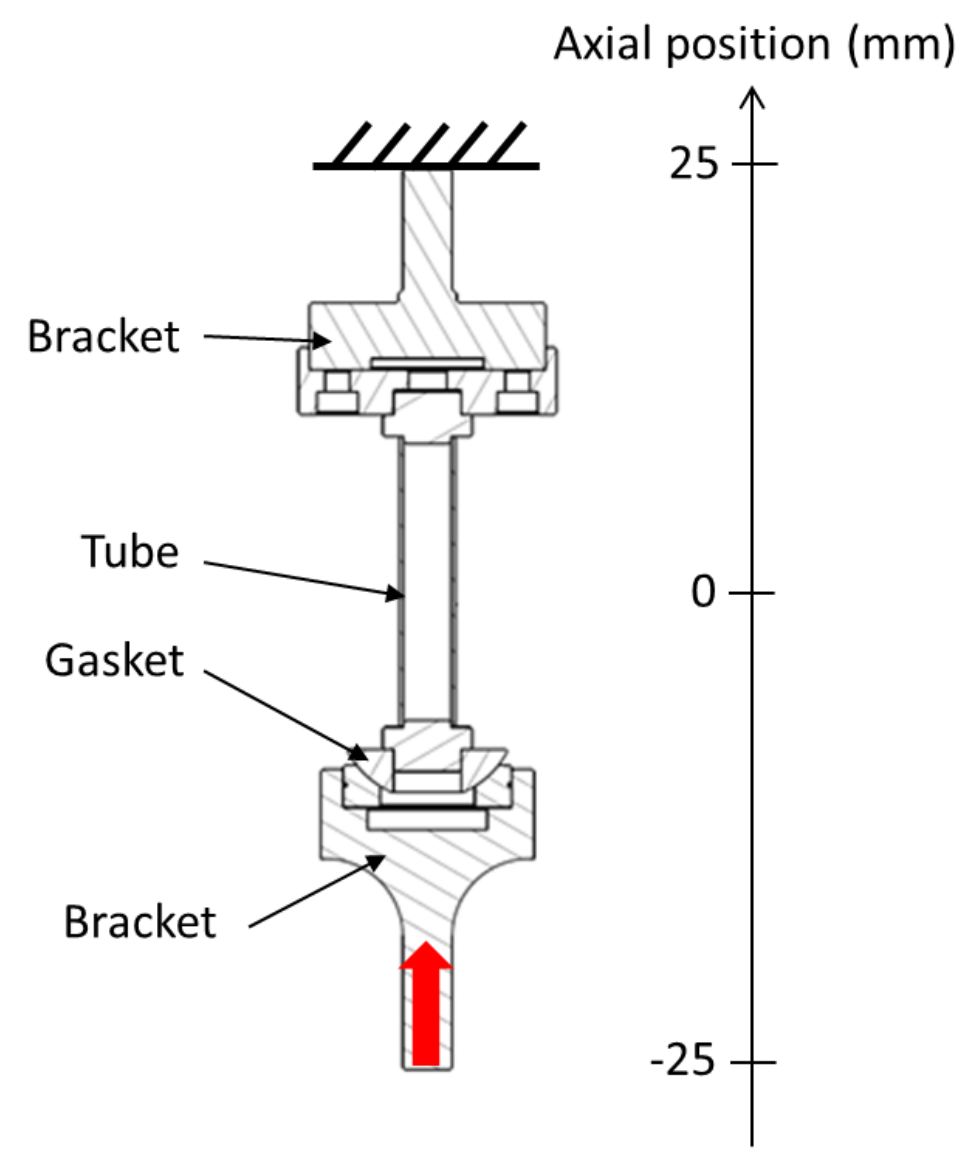

Figure 1: Experimental set-up

which giving rise to identical results, whatever the length of the specimen). The final deformed state (and thus the buckling phenomenon brought into play) is found to be axisymmetric, as expected for such a radius-to-thickness ratio. Moreover, Figure 4 depicts the longitudinal strain distribution throughout the specimen at a particular loading level (around the critical value). It confirms the fact that the deformations remain axisymmetric and that the buckling mode (namely the immediate post-critical deformed shape) displays an oscillatory pattern with variable amplitude, compliant with the sinusoidal damped mode obtained analytically. 


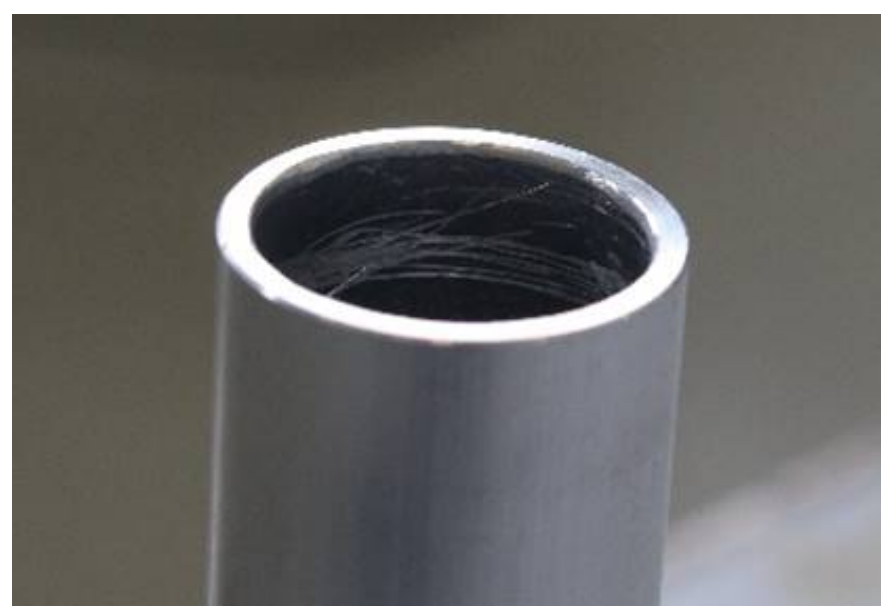

Figure 2: Geometry of the cylindrical tube

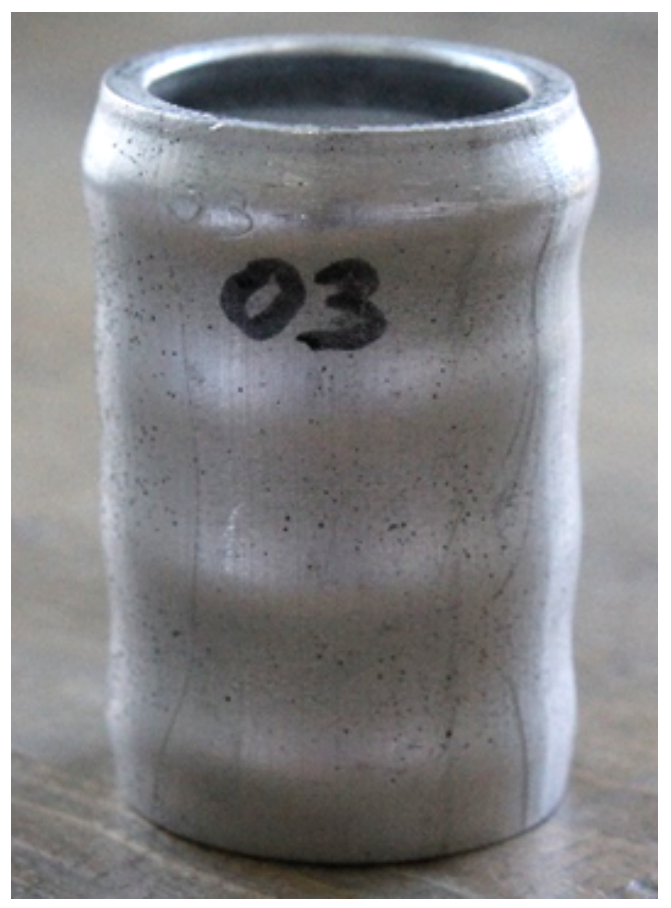

Figure 3: Post-buckled deformed shape of a specimen

\subsection{Tensile tests}

Tensile tests have also been performed on similar tubes in order to calibrate the constitutive law. Longer tubes are considered here (with $L=130 \mathrm{~mm}$ ) and special caps are positioned at both ends so as to reduce flattening effects due to hydraulic jaws at the maximum. Tests are conducted with a displacement speed of $0.5 \mathrm{~mm} / \mathrm{min}$, what roughly corresponds to a strain rate of $10^{-4} \mathrm{~s}^{-1}$. Finally, use is made of an extensometer so as to measure the longitudinal displacements in the tube during the tests and the load cell allows one to 


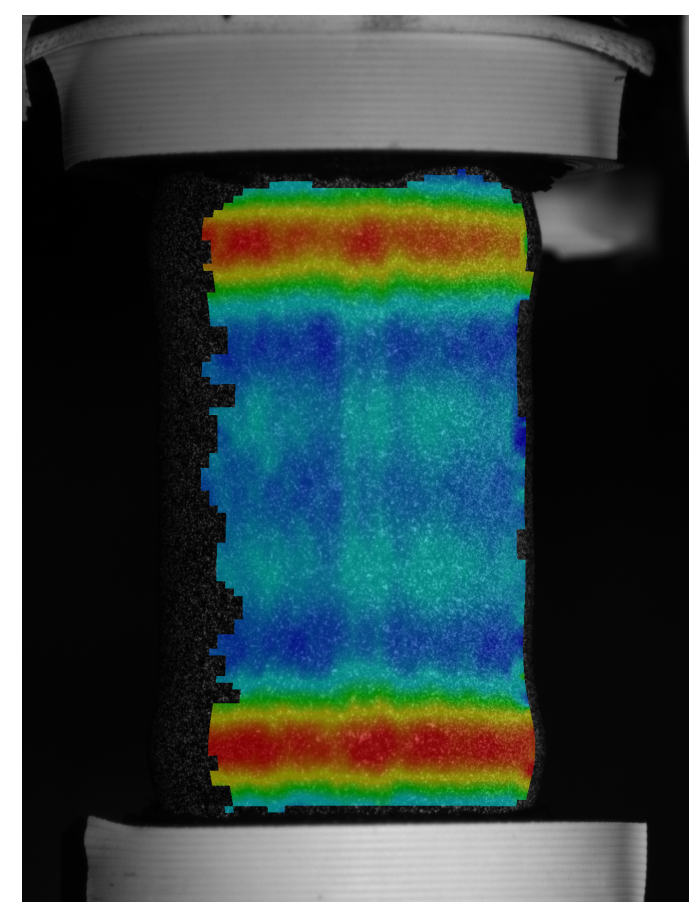

Figure 4: Strain mapping during the compression test

measure the tensile force at each time.

From the uniaxial tensile tests (carried out on the 6060 aluminum alloy to be characterized), one can plot the nominal uniaxial stress versus the nominal longitudinal strain, deriving straight from the axial force and displacement, respectively. The nominal stress-strain curve is transformed here into the true stress-strain curve (Cauchy stress versus Hencky logarithmic strain) so as to be implemented properly in Abaqus (which is formulated in terms of true stresses and strains). The choice of an elastoplastic constitutive law is then retained. The elastic response is supposed to be linear and isotropic, thus governed by the Hooke's law. The actual value of Young's modulus is obtained through a linear regression of the first part of the stress-strain curve (see Figure 5) and a standard value (for aluminum alloys) is retained for the Poisson's ratio. The plastic behavior is supposed to be governed by the von Mises yield criterion together with a non-linear isotropic hardening. Again, the hardening law is fitted from the true stress-(equivalent) plastic strain curve, using appropriately a quadratic expression, as seen in Figure 5.

The material properties are listed in Table 1.

\subsection{Numerical modeling with Abaqus software}

A numerical model of a cylindrical tube under axial compression is then developed, using Abaqus software, in order to correlate experimental and numerical results. The same geometric parameters as in compression 


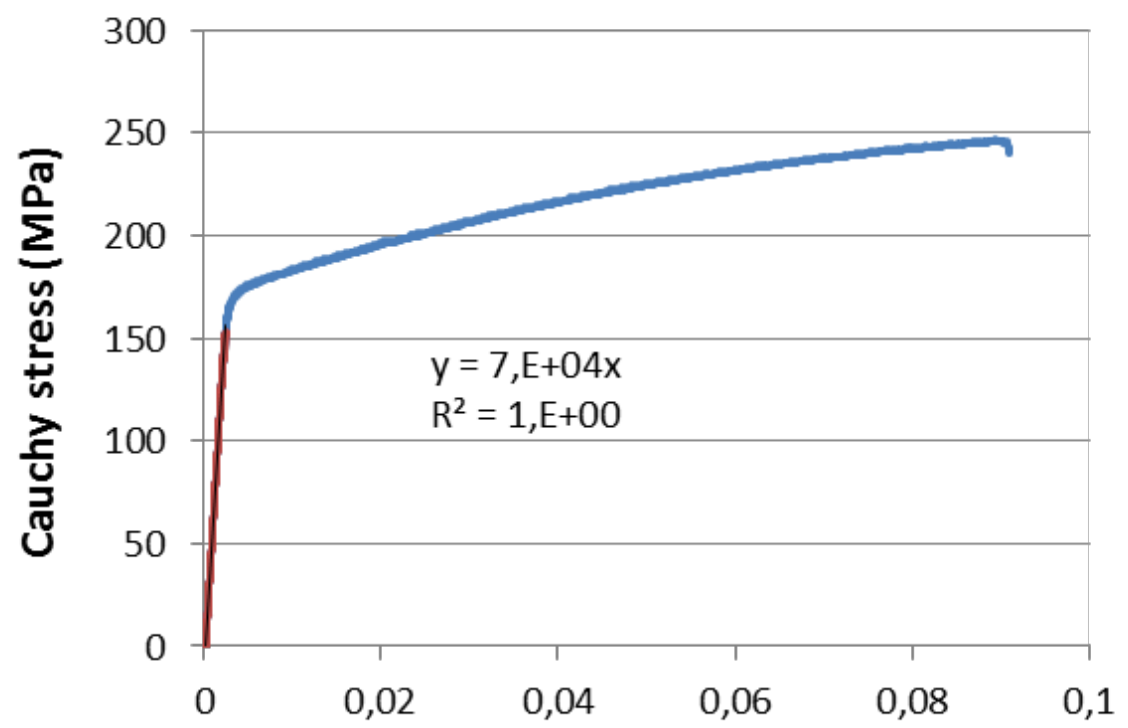

Hencky strain (-)

(a) Calibration of the Young's modulus

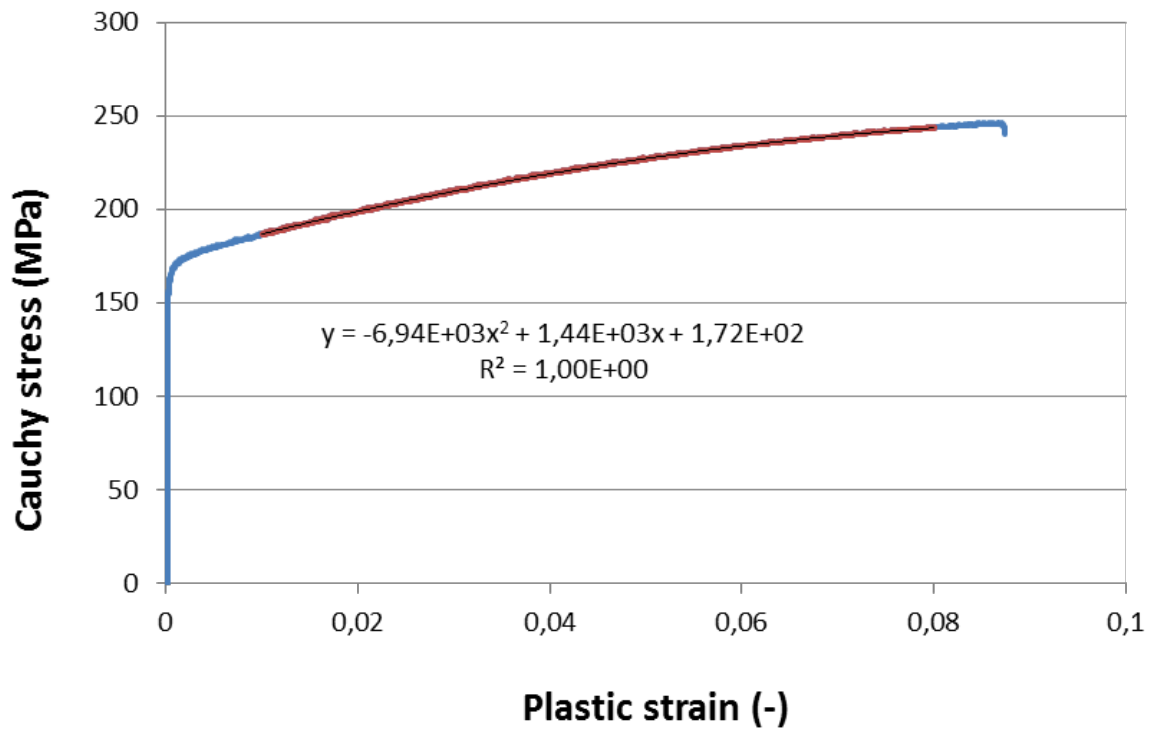

(b) Calibration of the hardening curve

Figure 5: Calibration of the elastoplastic constitutive law

experiments are naturally considered (they are recalled in Table 2).

Owing to the axisymmetric deformations observed in experiments, an axisymmetric shell model is retained. In practice, only a generating line of the cylinder is modeled using 1D shell axisymmetric quadratic 


\begin{tabular}{|c|c|c|c|}
\hline $\begin{array}{c}\text { Young's } \\
\text { modulus } E\end{array}$ & $\begin{array}{c}\text { Poisson's } \\
\text { ratio } \nu\end{array}$ & $\begin{array}{c}\text { Yield } \\
\text { stress } \sigma_{0}\end{array}$ & $\begin{array}{c}\text { Hardening } \\
\text { law } \sigma(p)\end{array}$ \\
\hline $70000 M P a$ & 0.33 & $172 M P a$ & $-6940 p^{2}+1440 p+172(M P a)$ \\
\hline
\end{tabular}

Table 1: Material properties used in numerical computations (Abaqus)

\begin{tabular}{|c|c|c|}
\hline Radius $R$ & Thickness $t$ & Length $L$ \\
\hline $11.5 \mathrm{~mm}$ & $2 \mathrm{~mm}$ & $50 \mathrm{~mm}$ or $75 \mathrm{~mm}$ \\
\hline
\end{tabular}

Table 2: Geometric parameters in numerical computations

finite elements (SAX2 elements in Abaqus). A short mesh convergence analysis led to the choice of 100 elements along the length of the tube and 5 Gaussian points are used in the shell thickness direction for the numerical integration. The previous elastoplastic constitutive law is implemented in Abaqus and computations are performed in the context of finite transformations. Use is made of Riks' algorithm (arc-length method) so as to handle automatically possible limit points encountered during the incremental calculations. Both edges of the generator are left free, except from the longitudinal displacement of one of them which is prevented so as to avoid any rigid mode, whereas the other edge is submitted to a longitudinal compressive force. With such a perfect model (without any geometric imperfection and any influence of the boundary conditions in the pre-critical stage), a small perturbation is needed so as to trigger the buckling phenomenon. Here, two infinitesimal radial centripetal forces are applied to the endpoints so as to get preferentially a buckling mode/post-buckling deformed shape with the edges turning inward, as observed in experiments (a specific value of $10^{-3} \mathrm{~N}$ has been arbitrarily retained for each force, noting that the buckling response does not depend on the particular value of the forces, as soon as they remain in this order of magnitude, namely negligible when compared to the compressive force).

\subsection{Numerical results and validation}

First, in Figure 6, the successive deformed shapes of the cylindrical tube obtained through the numerical incremental calculations are depicted and compared to their experimental counterparts observed during an arbitrary experiment (with a specimen length of $50 \mathrm{~mm}$ ) representative of all compression tests. The axial position (in abscissa) refers to the vertical axis in Figure 1 and the plotted curves correspond to the evolution of the external radius of the cylinder along the length of the tube, from the beginning of the test (blue curves) until the end (red curves). In both experimental and numerical cases, both edges move radially due to the absence of restraints and in accordance with the requested free edge boundary conditions, towards the outside during the pre-critical stage (as long as the deformations remain homogeneous along the tube) and then inwards when buckling occurs. The experimental and numerical post-critical deformed shapes are 
quite similar, even if the two ripples observed experimentally in the center of the specimen are not retrieved numerically.

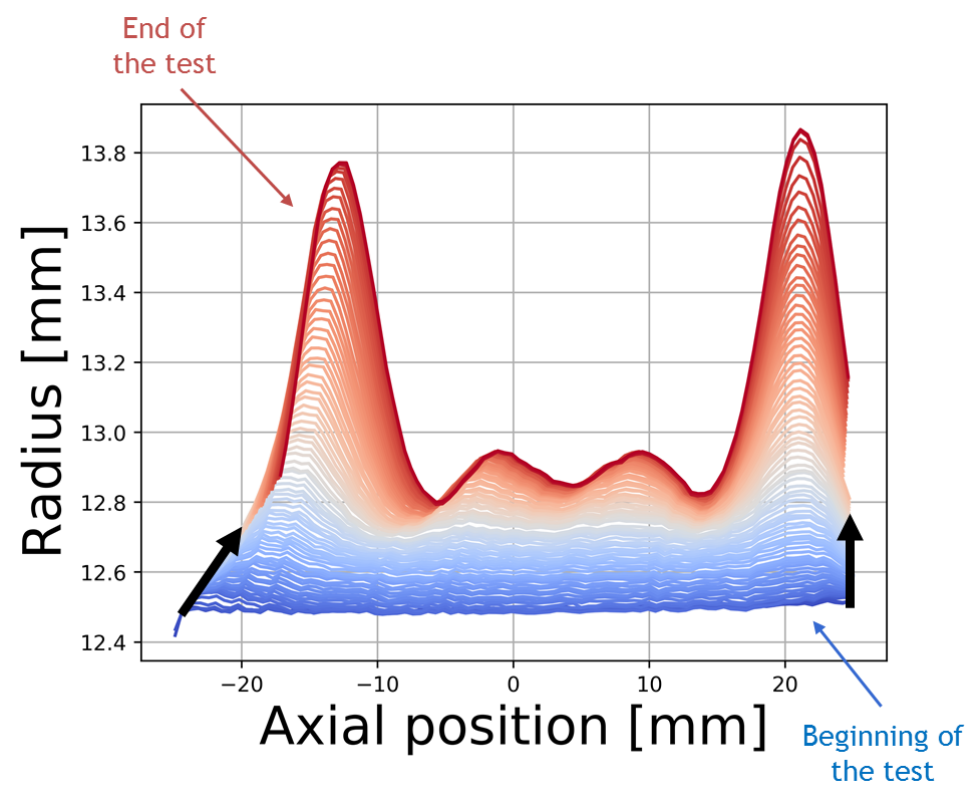

(a) Experimental results

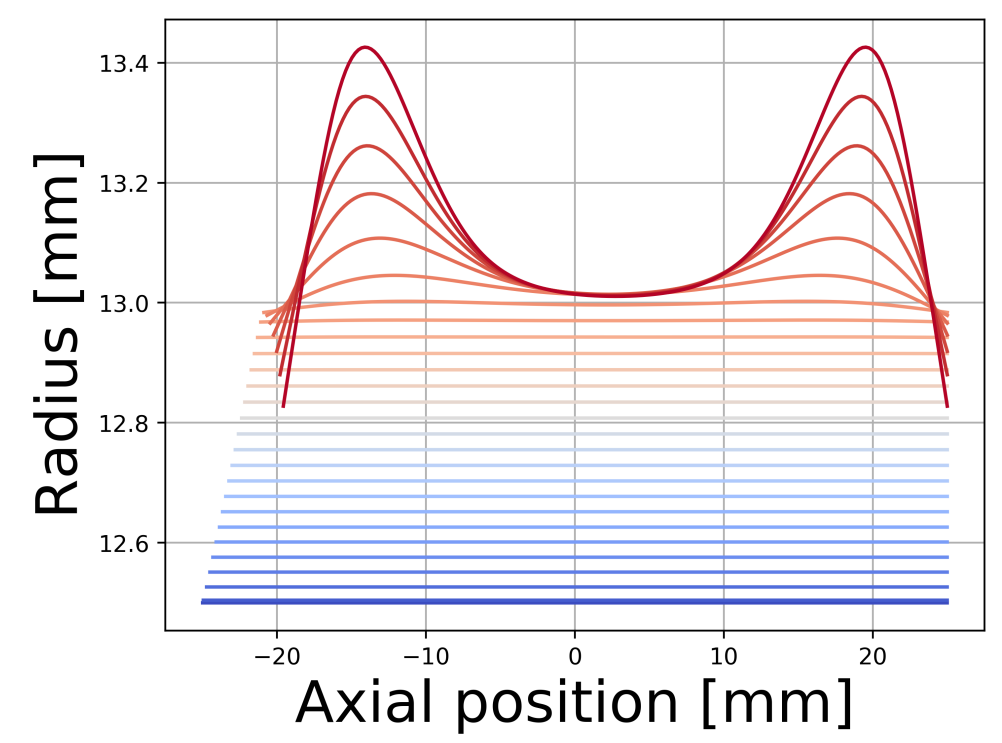

(b) Numerical deformed shapes with free edge boundary conditions

Figure 6: Evolution of the radius profile along the length of a specimen $(L=50 \mathrm{~mm})$

Above all, experimental and numerical nominal stress-strain curves (corresponding, this time, to a specimen of length $75 \mathrm{~mm}$ ) are plotted and compared to each other in Figure8 (let us mention that the nominal 
stress is none other than the axial compressive load divided by the cross-section area in the reference configuration, and the nominal strain is, in turn, proportional to the axial shortening). For comparison purposes, numerical computations have also been carried out in the cases of simply-supported and built-in edges (see Figure 7), and the corresponding stress-strain curves have also been plotted in Figure 8.

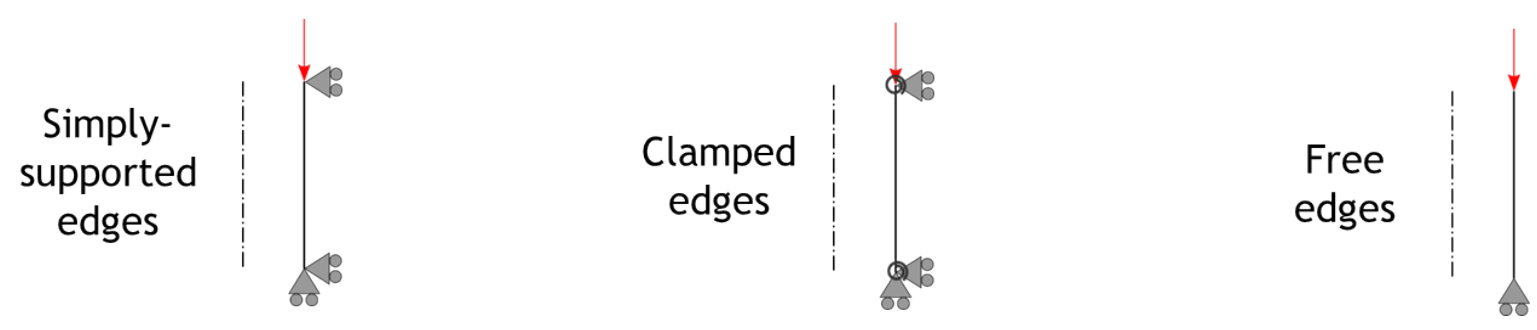

Figure 7: Different boundary conditions

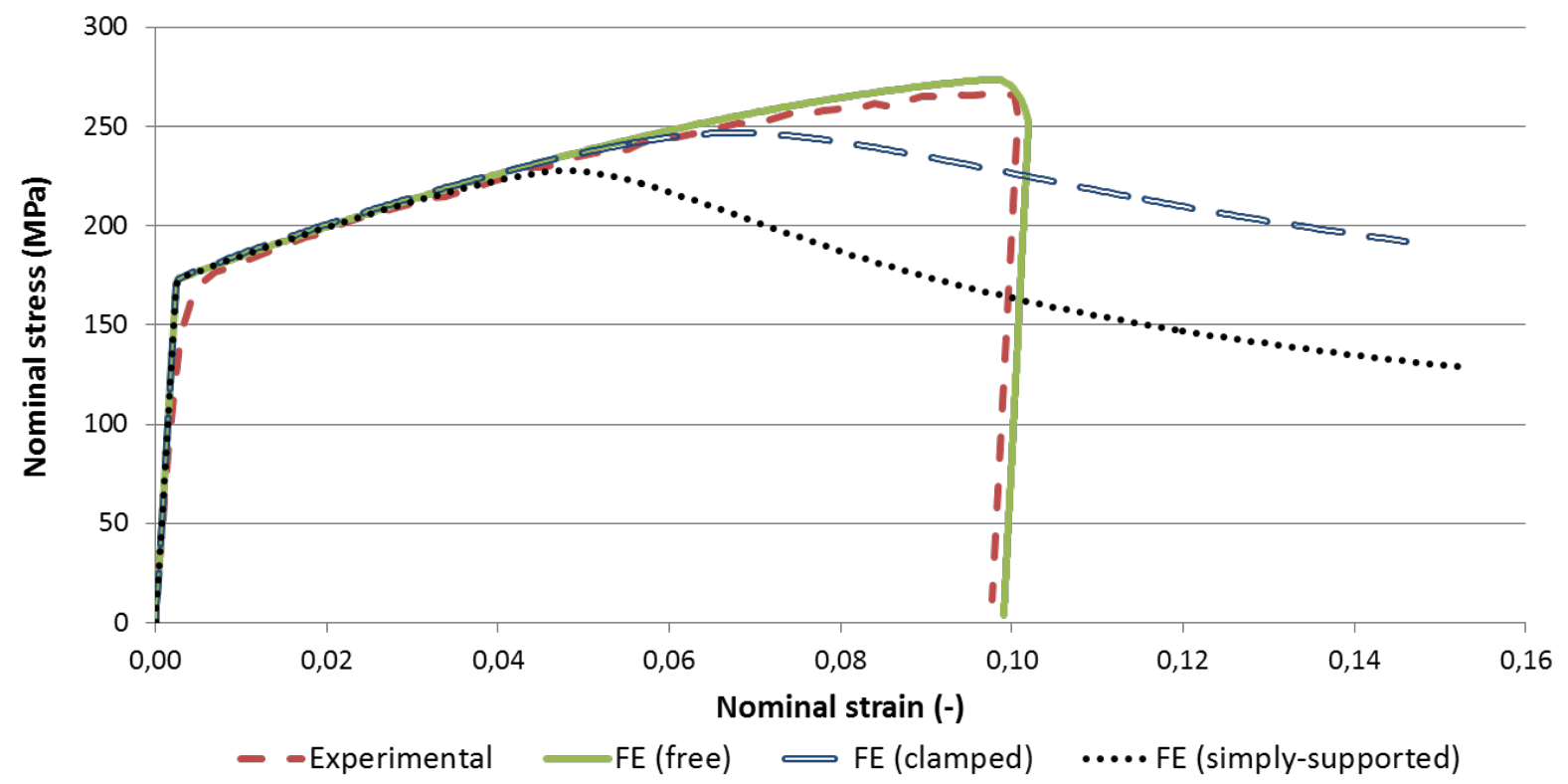

Figure 8: Comparison between experimental and numerical stress-strain curves of cylindrical tubes under axial compression with various boundary conditions $(L=75 \mathrm{~mm})$

All the curves are nearly coincident before buckling, namely during the pre-critical stage. Despite the heterogeneous pre-critical deformations naturally encountered in the two simply-supported and built-in cases, the pre-buckling responses defined as before in terms of average nominal stresses and strains coincide for the three boundary conditions, with an elastic stage followed by a plastic one (buckling is then supposed to occur in the plastic range at the tangent modulus critical load). Besides, three different critical loads are obtained numerically, depending on the boundary conditions. In the simply-supported and built-in cases, 
the bifurcation points (which can be roughly represented by the limit points) should be expected to be quite similar to each other and twice as high as the one corresponding to the free edges, according to the classical analytical critical values. However, in these two cases, due to edge effects (which are all the more strong in plasticity), the buckling problem is degenerated and the limit loads are more than halved in comparison with the theoretical value. Conversely, the limit point obtained numerically with free edges is in very good agreement with its experimental counterpart. It means that not only the force but also the axial shortening are quite the same, which confirms that the previous experimental tests reflect the free edge boundary conditions very well and that the unavoidable geometric imperfections in practice have here virtually no influence on the buckling response.

This perfect match between the two curves turns out to be a very interesting result which encourages us to investigate this problem analytically. The next section is thus devoted to the development of an analytical solution for the plastic buckling problem of a thick cylindrical shell under axial compression in the special case of free edges.

\section{Analytical study}

\subsection{Plastic bifurcation theory}

The subsequent developments rely on the plastic bifurcation theory whose main issues have already been presented in details in [24]. The most significant and useful results will be recalled here for completeness purposes.

The following plastic buckling analyses are conducted, as usually, by means of a total Lagrangian formulation, in the framework of the generalized standard materials theory [25, 26]. As strains remain moderate in the present study, the Green strain tensor $\mathbf{E}$ can be additively split in its elastic and plastic parts $\mathbf{E}^{e}$ and $\mathbf{E}^{p}$, respectively, which leads next to expressions analogous to those classically obtained in small strains [27, 28.

The elastic response of the material is supposed to be isotropic and is thus represented by the SaintVenant-Kirchhoff law involving the fourth-order elasticity tensor $\mathbf{D}$ whose components in an orthonormal basis are $D_{i j k l}=\lambda \delta_{i j} \delta_{k l}+\mu\left(\delta_{i k} \delta_{j l}+\delta_{i l} \delta_{k j}\right)$, where $\delta_{i j}$ is the Kronecker symbol and $\lambda$ and $\mu$ are the Lamé constants. Use will also be made of Young's modulus $E$ and Poisson's ratio $\nu$ which are related to $\lambda$ and $\mu$ by $\lambda=\frac{E \nu}{(1+\nu)(1-2 \nu)}$ and $\mu=\frac{E}{2(1+\nu)}$.

In the framework of the $J_{2}$ flow theory of plasticity, the elastic region is defined by the von Mises yield function with an isotropic hardening:

$$
f(\boldsymbol{\Sigma}, A)=\sqrt{\frac{3}{2} \boldsymbol{\Sigma}^{d}: \boldsymbol{\Sigma}^{d}}-\sigma_{0}-A
$$

where $\boldsymbol{\Sigma}^{d}$ means the deviatoric part of the second Kirchhoff stress tensor $\boldsymbol{\Sigma}, \sigma_{0}$ is the initial yield stress and $A$ the thermodynamical force conjugate to the isotropic hardening parameter $a$. 
The normality rules state that the plastic strain $\mathbf{E}^{p}$ as well as the hardening variable $a$ derive from a dissipative potential which is equal to the yield function:

$$
\dot{\mathbf{E}}^{p}=\dot{\Lambda} \frac{\partial f}{\partial \boldsymbol{\Sigma}} \quad \dot{a}=-\dot{\Lambda} \frac{\partial f}{\partial A}=\dot{\Lambda}
$$

where $\dot{\Lambda}$ is the plastic multiplier, whose expression can be deduced from the consistency condition.

The material tangent elastoplastic tensor takes then the following form:

$$
\mathbf{D}^{p}=\frac{\partial \boldsymbol{\Sigma}}{\partial \mathbf{E}}=\mathbf{D}-\frac{\mathbf{D}: \frac{\partial f}{\partial \boldsymbol{\Sigma}} \otimes \frac{\partial f}{\partial \boldsymbol{\Sigma}}: \mathbf{D}}{H+\frac{\partial f}{\partial \boldsymbol{\Sigma}}: \mathbf{D}: \frac{\partial f}{\partial \boldsymbol{\Sigma}}}
$$

where $H=\frac{\partial A}{\partial a}$ is the current hardening modulus.

Finally, the nominal tangent elastoplastic tensor writes:

$$
\mathbf{K}^{p}=\frac{\partial \boldsymbol{\Pi}}{\partial \mathbf{F}}=\mathbf{F} \cdot \frac{\partial \boldsymbol{\Sigma}}{\partial \mathbf{E}} \cdot \mathbf{F}^{T}+(\mathbb{I} \cdot \boldsymbol{\Sigma})^{T}
$$

where $\mathbf{F}$ stands for the deformation gradient, $\boldsymbol{\Pi}=\mathbf{F} . \boldsymbol{\Sigma}$ the first Kirchhoff stress tensor, $\mathbb{I}$ represents the fourth-order unit tensor $\left(\mathbb{I}_{i j k l}=\delta_{i l} \delta_{k j}\right)$ and the superscript ${ }^{T}$ the transposition of a second-order tensor or the major transposition of a fourth-order tensor.

Assuming now that (i) the buckling phenomenon occurs in the plastic regime (the whole solid is assumed to be plastified on the fundamental branch at critical time) and that (ii) the bifurcation takes place at the tangent modulus critical load (with incipient unloading), the critical load $\xi_{c}$ and the bifurcation mode $\mathbf{X}$ of an arbitrary $3 \mathrm{D}$ body $\Omega$ can be obtained by solving the following bifurcation equation [29, 30]:

$$
\forall \delta \mathbf{U}, \quad \int_{\Omega} \nabla^{T} \delta \mathbf{U}: \mathbf{K}^{p}\left(\xi_{c}\right): \nabla \mathbf{X} \quad d \Omega=0
$$

where $\delta \mathbf{U}$ can be considered as a test function or, more physically, as the virtual variation of the unknown displacement field $\mathbf{U}$.

Let $\left(\mathbf{e}_{1}, \mathbf{e}_{2}, \mathbf{e}_{3}\right)$ be a fixed orthonormal basis and let us consider the particular case of a compressive load in the $\mathbf{e}_{1}$-direction which gives rise to a uniaxial nominal stress state as follows:

$$
\boldsymbol{\Pi}=-\xi \mathbf{e}_{1} \otimes \mathbf{e}_{1} \quad(\xi>0)
$$

In case of small pre-critical deformations $(\|\nabla \mathbf{U}\| \ll 1)$, the second Kirchhoff stress tensor $\boldsymbol{\Sigma}$ coincides with the previous one. Hence, the material tangent elastoplastic tensor in Equation (4) reads:

$$
\mathbf{D}^{p}=\mathbf{D}-\frac{\mu^{2}}{H+3 \mu}\left[\mathbf{I}-3 \mathbf{e}_{1} \otimes \mathbf{e}_{1}\right] \otimes\left[\mathbf{I}-3 \mathbf{e}_{1} \otimes \mathbf{e}_{1}\right]
$$

and the nominal tangent elastoplastic tensor in Equation (5) becomes:

$$
\mathbf{K}^{p} \approx \frac{\partial \boldsymbol{\Sigma}}{\partial \mathbf{E}}+(\mathbb{I} . \boldsymbol{\Sigma})^{T}=\mathbf{D}^{p}-\xi \mathbf{e}_{i} \otimes \mathbf{e}_{1} \otimes \mathbf{e}_{1} \otimes \mathbf{e}_{i}
$$


where implicit summations are made on repeated indices.

Furthermore, when dealing with 2D models like shells, the plane stress condition must be verified. Enforcing a null normal material stress in the thickness direction $\left(\Sigma_{33}=0\right)$ leads one to replace tensor $\mathbf{D}^{p}$ in (9) with the suitable reduced tensor $\mathbf{C}^{p}$, whose components in the orthonormal basis $\left(\mathbf{e}_{1}, \mathbf{e}_{2}, \mathbf{e}_{3}\right)$ are:

$$
C_{i j k l}^{p}=D_{i j k l}^{p}-\frac{D_{i j 33}^{p} D_{33 k l}^{p}}{D_{3333}^{p}} \quad(i, j) \neq(3,3),(k, l) \neq(3,3)
$$

The tensor $\mathbf{C}^{p}$ has the major and both minor symmetries $\left(C_{i j k l}^{p}=C_{k l i j}^{p}=C_{j i k l}^{p}=C_{i j l k}^{p}\right)$, and the following notations will be further used for brevity (the other components being zero):

$$
\begin{aligned}
& \alpha=C_{1111}^{p}=E \frac{1+3 \frac{E_{T}}{E}}{5-4 \nu-(1-2 \nu)^{2} \frac{E_{T}}{E}} \\
& \beta=C_{1122}^{p}=E \frac{2-2(1-2 \nu) \frac{E_{T}}{E}}{5-4 \nu-(1-2 \nu)^{2} \frac{E_{T}}{E}} \\
& \gamma=C_{2222}^{p}=E \frac{4}{5-4 \nu-(1-2 \nu)^{2} \frac{E_{T}}{E}}
\end{aligned}
$$

where the tangent modulus $E_{T}$ is related to the Young's modulus $E$ and the isotropic hardening modulus $H$ by $\frac{1}{E_{T}}=\frac{1}{E}+\frac{1}{H}$.

Finally, in a uniaxial stress state and under the plane stress condition, the bifurcation equation (6) writes:

$$
\forall \delta \mathbf{U}, \quad \int_{\Omega} \nabla^{T} \delta \mathbf{U}:\left(\mathbf{C}^{p}-\xi \mathbf{e}_{i} \otimes \mathbf{e}_{1} \otimes \mathbf{e}_{1} \otimes \mathbf{e}_{i}\right): \nabla \mathbf{X} \quad d \Omega=0
$$

\subsection{Cylindrical tube under axial compression}

The general results above will henceforth be applied to the particular case of a cylinder under axial compression. First, using standard kinematics and conventional hypotheses, the classical solution will be naturally found. On this basis, other solutions will be investigated so as to better agree with previous experimental and numerical results.

Let us consider a cylindrical tube defined in the reference configuration by the length $L$ along the $\mathbf{e}_{x}$-axis, the average radius $R$ and the thickness $t$, loaded by a uniformly distributed nominal axial compressive stress $\Pi_{x x}=-\xi<0$. The tube is made of an elastoplastic material with an isotropic hardening and the yield stress $\sigma_{0}$ is assumed small enough for the plastic strains to appear before buckling.

In the first instance, the kinematics is described by the Koiter model, equivalent to the Love-Kirchhoff plate model. In the axisymmetric framework, there is no circumferential displacement and nothing depends on the circumferential coordinate. The displacement field of the middle surface of the shell is thus defined by the two following components: $U(x)$ (axial) and $W(x)$ (radial). Consider a current particle in the cylinder, whose position in the reference configuration is defined by $x$ and the thickness parameter $\rho\left(-\frac{t}{2} \leqslant \rho \leqslant \frac{t}{2}\right)$. 
Its displacement can be expressed in the local cylindrical basis $\left(\mathbf{e}_{x}, \mathbf{e}_{\theta}, \mathbf{e}_{r}\right)$ as:

$$
\mathbf{U}=\mid \begin{aligned}
& U-\rho W,,_{x} \\
& 0 \\
& W
\end{aligned}
$$

The bifurcation mode $\mathbf{X}$ is naturally expressed according to the same kinematics:

$$
\mathbf{X}=\mid \begin{aligned}
& \mathcal{U}-\rho \mathcal{W},_{x} \\
& 0 \\
& \mathcal{W}
\end{aligned}
$$

whence the bifurcation mode gradient:

$$
\nabla \mathbf{X}=\left[\begin{array}{ccc}
\mathcal{U},_{x}-\rho \mathcal{W},_{x x} & 0 & -\mathcal{W},_{x} \\
0 & \frac{\mathcal{W}}{R} & 0 \\
\mathcal{W},_{x} & 0 & 0
\end{array}\right]
$$

One has then to integrate the bifurcation equation 12 in the shell thickness and then adequately integrate by parts with respect to $x$. Without going into details, the two resulting local equations arise:

$$
\left\{\begin{array}{l}
\left(\alpha-\xi_{c}\right) \mathcal{U},_{x x}+\frac{\beta}{R} \mathcal{W},_{x}=0 \\
\frac{\beta}{R} \mathcal{U},_{x}+\frac{\gamma}{R^{2}} \mathcal{W}+\xi_{c} \mathcal{W},_{x x}+\left(\alpha-\xi_{c}\right) \frac{t^{2}}{12} \mathcal{W},_{x x x x}=0
\end{array}\right.
$$

together with the natural boundary conditions, which will be specified later.

Since it is difficult to determine the bifurcation mode in the case of a free edge cylinder with finite length, we limit consideration to a cylinder of infinite length. The symmetry makes it possible to study one half of the cylinder only, with the free edge at $x=0$ and the plane of symmetry put at infinity.

Displacement boundary conditions are enforced at infinity - namely the displacements $\mathcal{U}$ and $\mathcal{W}$ and their derivatives with respect to $x$ are assumed to be zero. Conversely, at the edge $x=0$, the axial displacement is null $(\mathcal{U}(0)=0)$, whereas the displacement $\mathcal{W}$ and its derivative with respect to $x$ are free, in such a way that the corresponding natural boundary conditions (deriving from the previous integration by parts) must be verified:

$$
\left\{\begin{array}{l}
\mathcal{W}_{x_{x x}}(0)=0 \\
\left(\alpha-\xi_{c}\right) \frac{t^{2}}{12} \mathcal{W}_{,_{x x x}}(0)+\xi_{c} \mathcal{W}_{,_{x}}(0)=0
\end{array}\right.
$$

In classical buckling analyses, one presupposes that pre-critical deformations are small. This amounts to saying that the sought critical stress is negligible in comparison to constitutive moduli. Here, taking into account that $\xi_{c} \ll \alpha$ allows one to rewrite Equations 16 and 177 in a simplified way:

$$
\left\{\begin{array}{l}
\alpha \mathcal{U},_{x x}+\frac{\beta}{R} \mathcal{W},_{x}=0 \\
\frac{\beta}{R} \mathcal{U},_{x}+\frac{\gamma}{R^{2}} \mathcal{W}+\xi_{c} \mathcal{W},_{x x}+\alpha \frac{t^{2}}{12} \mathcal{W}_{{ }_{x x x x}}=0
\end{array}\right.
$$


and:

$$
\left\{\begin{array}{l}
\mathcal{W},_{x x}(0)=0 \\
\alpha \frac{t^{2}}{12} \mathcal{W},_{x x x}(0)+\xi_{c} \mathcal{W},_{x}(0)=0
\end{array}\right.
$$

Combining the two relations of Equation (18) leads to a single equation involving the radial component of the eigenmode:

$$
\alpha \frac{t^{2}}{12} \mathcal{W},_{x x x x}+\xi_{c} \mathcal{W},_{x x}+\frac{1}{R^{2}}\left(\gamma-\frac{\beta^{2}}{\alpha}\right) \mathcal{W}=0
$$

Expression (20), valid for plastic buckling, is similar to the simplified form (in the axisymmetric case) of the Donnell equation [31] for the well-known elastic buckling of a cylinder under axial compression (see also 32 ).

The axisymmetric buckling mode is then assumed to be a sinusoidal function weighted by a decreasing exponential function:

$$
\mathcal{W}=\left(k_{1} \cos (\eta x)+k_{2} \sin (\eta x)\right) \exp (-\zeta x)
$$

where $k_{1}, k_{2}, \eta$ and $\zeta$ are to be determined. Relation 21) expresses the fact that the radial displacement is significant at the end of the cylinder and is zero in the plane of symmetry.

By inserting Equation 21] in (20), one obtains:

$$
\left\{\begin{array}{c}
\alpha \frac{t^{2}}{12}\left(k_{1} \eta^{4}+4 k_{2} \eta^{3} \zeta-6 k_{1} \eta^{2} \zeta^{2}-4 k_{2} \eta \zeta^{3}+k_{1} \zeta^{4}\right) \\
+\xi_{c}\left(-k_{1} \eta^{2}-2 k_{2} \eta \zeta+k_{1} \zeta^{2}\right)+\left(\gamma-\frac{\beta^{2}}{\alpha}\right) \frac{k_{1}}{R^{2}}=0 \\
\alpha \frac{t^{2}}{12}\left(k_{2} \eta^{4}-4 k_{1} \eta^{3} \zeta-6 k_{2} \eta^{2} \zeta^{2}+4 k_{1} \eta \zeta^{3}+k_{2} \zeta^{4}\right) \\
+\xi_{c}\left(-k_{2} \eta^{2}+2 k_{1} \eta \zeta+k_{2} \zeta^{2}\right)+\left(\gamma-\frac{\beta^{2}}{\alpha}\right) \frac{k_{2}}{R^{2}}=0
\end{array}\right.
$$

The boundary conditions at infinity result in the single condition $\zeta>0$. The natural conditions 19 lead to:

$$
\left\{\begin{array}{l}
-k_{1} \eta^{2}-2 k_{2} \eta \zeta+k_{1} \zeta^{2}=0 \\
\alpha \frac{t^{2}}{12}\left(-k_{2} \eta^{3}+3 k_{1} \eta^{2} \zeta+3 k_{2} \eta \zeta^{2}-k_{1} \zeta^{3}\right)+\xi_{c}\left(k_{2} \eta-k_{1} \zeta\right)=0
\end{array}\right.
$$

The bifurcation mode is defined within a factor which can be determined by enforcing $k_{2}=-t$. The four equations 22,23$)$ then give the critical stress:

$$
\xi_{c}=\frac{t}{6 R} \sqrt{3\left(\alpha \gamma-\beta^{2}\right)}=\frac{E t}{R} \sqrt{\frac{\frac{E_{T}}{E}}{3\left[5-4 \nu-(1-2 \nu)^{2} \frac{E_{T}}{E}\right]}}
$$

and:

$$
\begin{aligned}
& k_{1}=t \sqrt{3} \\
& \eta=\frac{s}{\sqrt{R t}} \quad\left(s=\sqrt[4]{\frac{27\left[5-4 \nu-(1-2 \nu)^{2} \frac{E_{T}}{E}\right] \frac{E_{T}}{E}}{\left(1+3 \frac{E_{T}}{E}\right)^{2}}}\right) \\
& \zeta=\frac{s}{\sqrt{3 R t}}
\end{aligned}
$$


The complete eigenmode is finally obtained by solving Equation (18) with the remaining boundary conditions:

$$
\left\{\begin{array}{l}
\mathcal{U}=-t \sqrt{\frac{3 t}{R}} \frac{q}{s} \sin \left(\frac{s x}{\sqrt{R t}}\right) \exp \left(-\frac{s x}{\sqrt{3 R t}}\right) \\
\mathcal{V}=0 \\
\mathcal{W}=t\left[\sqrt{3} \cos \left(\frac{s x}{\sqrt{R t}}\right)-\sin \left(\frac{s x}{\sqrt{R t}}\right)\right] \exp \left(-\frac{s x}{\sqrt{3 R t}}\right)
\end{array}\right.
$$

where the mode has been normalized so that $\mathcal{W}(0)=t \sqrt{3}$ and:

$$
q=2 \frac{1-(1-2 \nu) \frac{E_{T}}{E}}{1+3 \frac{E_{T}}{E}}
$$

The previous solution (Equations 24 260) coincides with the well-known one of Batterman [17] and has already been found by one of the authors in 24]. It has also been validated by comparison to numerical computations, but always in the case of thin shells with particularly high hardening moduli 33 . In the present case of a thicker shell with a lower (realistic) hardening modulus, there is a large discrepancy between the classical analytical solution and the numerical/experimental results (namely a relative error around 20$25 \%$ ). The objective of the present study is thus to improve the analytical model by considering more general hypotheses so as to compare better with the previous numerical and experimental solutions. On one hand, the use of new kinematics (involving transverse shear effects) in the analytical formulation will be investigated and, on the other hand, geometric non-linearities will be taken into account in the model. For conciseness purposes, the critical values for the problem in hand, obtained successively with the different analytical models considered in the sequel, will be reported at the end of the paper.

\subsection{Influence of transverse shear}

For simplicity purposes, the case of simply-supported edges will be considered here, for which the classical plastic buckling stress (without considering transverse shear effects) is twice the critical stress obtained above in the free edge condition (see Equation (24)):

$$
\xi_{c}^{s s}=\frac{t}{3 R} \sqrt{3\left(\alpha \gamma-\beta^{2}\right)}
$$

Owing to the extremely low radius-to-thickness ratio encountered in such very thick cylindrical shells, an alternative analytical solution may be developed, considering Mindlin-Reissner type kinematics (based on the first-order shear deformation theory and taking into account therefore the transverse shear effects) instead of the previous Koiter kinematics. In the axisymmetric framework, the displacement field of the middle surface of the shell is now defined by the three following components: the longitudinal displacement $U(x)$, the radial displacement $W(x)$ and the rotation about the orthoradial direction $\theta(x)$. Consider a current particle in the cylinder, whose position in the reference configuration is defined by $x$ and the thickness parameter $\rho$ 
$\left(-\frac{t}{2} \leqslant \rho \leqslant \frac{t}{2}\right)$. Its displacement can be thus expressed in the local cylindrical basis $\left(\mathbf{e}_{x}, \mathbf{e}_{\theta}, \mathbf{e}_{r}\right)$ as:

$$
\mathbf{U}=\mid \begin{aligned}
& U-\rho \theta \\
& 0 \\
& W
\end{aligned}
$$

The bifurcation mode $\mathbf{X}$ is naturally expressed according to the same kinematics:

$$
\mathbf{X}=\mid \begin{aligned}
& \mathcal{U}-\rho \Theta \\
& 0 \\
& \mathcal{W}
\end{aligned}
$$

whence the bifurcation mode gradient:

$$
\nabla \mathbf{X}=\left[\begin{array}{ccc}
\mathcal{U},_{x}-\rho \Theta,,_{x} & 0 & -\Theta \\
0 & \frac{\mathcal{W}}{R} & 0 \\
\mathcal{W},_{x} & 0 & 0
\end{array}\right]
$$

Integrating the bifurcation equation 12 in the shell thickness, then integrating by parts with respect to $x$ and eliminating negligible terms give rise to the three resulting local equations:

$$
\left\{\begin{array}{l}
\alpha \mathcal{U}_{{ }_{x x}}+\frac{\beta}{R} \mathcal{W},_{x}=0 \\
\frac{\beta}{R} \mathcal{U},_{x}+\frac{\gamma}{R^{2}} \mathcal{W}-k \mu \mathcal{W},_{x x}+k \mu \Theta,_{x}+\xi_{c}^{T S} \mathcal{W},_{x x}=0 \\
\alpha \frac{t^{2}}{12} \Theta,_{x x}+k \mu \mathcal{W},_{x}-k \mu \Theta=0
\end{array}\right.
$$

together with the natural boundary conditions, which will be specified later. In Equation $32, k$ is the so-called shear correction factor involved in the first-order shear deformation theory and $\xi_{c}^{T S}$ represents the sought critical stress.

According to the simply-supported edges, the kinematical and natural boundary conditions write as follows:

$$
\left\{\begin{array}{l}
\mathcal{U}(0)=0 \\
\alpha \mathcal{U},{ }_{x}(L)+\frac{\beta}{R} \mathcal{W}(L)=0 \\
\mathcal{W}(0)=0 \\
\mathcal{W}(L)=0 \\
\Theta,{ }_{x}(0)=0 \\
\Theta,{ }_{x}(L)=0
\end{array}\right.
$$

A sinusoidal expression can be classically assumed for the radial component of the buckling mode (see 24. for more details):

$$
\mathcal{W}(x)=\sin \frac{n \pi x}{L}
$$


After a few calculations, the remaining components of the mode can be derived together with the critical stress, depending on the wave number $n$ :

$$
\xi_{c}^{n}=\frac{12 \alpha \gamma k \mu L^{4}-n^{2} \pi^{2} \alpha \beta^{2} L^{2} t^{2}+n^{4} \pi^{4} \alpha^{2} k \mu R^{2} t^{2}+n^{2} \pi^{2} \alpha^{2} \gamma L^{2} t^{2}-12 \beta^{2} k \mu L^{4}}{n^{2} \pi^{2} \alpha R^{2}\left(n^{2} \pi^{2} \alpha t^{2}+12 k \mu L^{2}\right)}
$$

Considering that $n$ is sufficiently high, one can find the first critical stress by minimizing the previous expression 35 with respect to $n \in \mathbb{R}$ :

$$
\xi_{c}^{T S}=\frac{3 t\left(\alpha \gamma-\beta^{2}\right)\left(t \sqrt{3\left(\alpha \gamma-\beta^{2}\right)}\left[k^{2} \mu^{2} R^{2}+\frac{t^{2}}{36}\left(\beta^{2}-\alpha \gamma\right)\right]+4 k^{3} \mu^{3} R^{3}\right)}{k \mu R^{2}\left(6 k \mu R+t \sqrt{3\left(\alpha \gamma-\beta^{2}\right)}\right)\left(t\left(\alpha \gamma-\beta^{2}\right)+2 k \mu R \sqrt{3\left(\alpha \gamma-\beta^{2}\right)}\right)}
$$

Considering the geometric and material parameters in hand in the present study, namely the values in Tables 1 and 2, the relative difference between $\xi_{c}^{T S}$ and $\xi_{c}^{s s}$ is less than $1 \%$. It means that, even for such extremely thick cylinders, the transverse shear effects remain negligible and are certainly not responsible for the mismatch between the classical analytical value and the experimental and numerical results, as far as plastic buckling is considered (in elasticity, the influence is much more important).

In view of this, transverse shear effects will be disregarded in the sequel and attention will be focused hereafter to the influence of geometric non-linearities, which may explain on their own the discrepancies between analytical and numerical critical values.

\subsection{Geometric non-linearities}

The use of the so-called bifurcation equation (6) provides a convenient framework for a buckling analysis since all the material and geometric non-linearities are compiled in the nominal tangent elastoplastic tensor $\mathbf{K}^{p}$ defined in its complete form in Equation (5), which allows one to deal easily with plastic buckling. In the previous classical solution procedure, the bifurcation equation has been linearized by assuming $\mathbf{F} \approx \mathbf{I}$ in $(5)$, thereby supposing that pre-critical deformations were infinitesimal. Next, the geometric stiffness operator $(\mathbb{I} . \boldsymbol{\Sigma})^{T}$ has been partially simplified, by neglecting the critical stress $\xi_{c}$ with respect to the reduced modulus $\alpha$ in Equations (16) and (17), thus keeping in both equations only the term $\xi_{c}$ responsible for the buckling phenomenon.

The first possible refinement of the previous solution consists in solving Equations (16) and (17) instead of Equations (18) and (19), considering the whole influence of pre-stresses on the current moduli. In doing so, one obtains a similar buckling mode and a new expression for the critical stress:

$$
\xi_{c}^{N L}=\frac{\left(\alpha \gamma t-2 \beta^{2} t+4 \alpha \sqrt{3\left[\left(\alpha \gamma-\beta^{2}\right) R^{2}+\frac{1}{48} \gamma^{2} t^{2}\right]}\right) t}{24 \alpha R^{2}+\gamma t^{2}+4 \sqrt{3\left[\left(\alpha \gamma-\beta^{2}\right) R^{2}+\frac{1}{48} \gamma^{2} t^{2}\right]} t}
$$

For validation purposes, a home-made finite element program (NLFEAS) is first used instead of Abaqus software for the two following reasons: (i) contrary to Abaqus, the finite element formulation in NLFEAS 
relies on a total Lagrangian framework, like the analytical formulation (it means particularly that the constitutive law is equally written in terms of the second Kirchhoff stress and Green strain tensors in both numerical and analytical models) and (ii) unlike Abaqus, NLFEAS has specific branching methods that enable it to capture the true bifurcation loads without the use of any imperfection.

A 2D axisymmetric finite element model is retained here in the context of finite plasticity. The axisymmetric shell is thus discretized in 2D isoparametric 8-node quadratic elements using 4 Gaussian points in each direction for the numerical integration. A few preliminary mesh convergence tests show that only one element through the thickness turns out to be sufficient. Loading and boundary conditions are applied consistently with the ones defined earlier in the axisymmetric shell model.

A few computations have been performed for various sets of geometric and material parameters and the following conclusions have been drawn. In any case, the immediate post-critical deformed shape obtained with the 2D numerical model is in very good agreement with the analytical modal shape, as illustrated in Figure 9. From a qualitative point of view, the bifurcation (which is accurately defined through the use of NLFEAS) comes just before the maximum load value reached in the post-buckling curve, as commonly observed in other numerical applications in the literature, whatever the geometry and material considered. The subsequent analysis will thus only focus on the critical stress which will be numerically identified to the limit stress. In the considered range of length-to-radius ratio, it has been checked that the critical stress hardly depends on the cylinder length as if it was of semi-infinite length.

The new critical value (Equation (37)) has been then validated using NLFEAS software. For simplicity purposes, a linear isotropic hardening has been considered, with various hardening moduli $H$. In each numerical computation, the yield stress $\sigma_{0}$ is arbitrarily chosen small enough so that plastification occurs before the tangent modulus critical load but also close enough from the critical stress (37) in order to limit plastic strains during the pre-critical stage and prevent voiding the small strain assumption.

The geometric and material parameters used for the present validation are quite similar to the previous experimental values, as shown in Tables 3 and 4 . The comparison between analytical and numerical results is depicted in Figure 10 .

\begin{tabular}{|c|c|c|}
\hline Radius $R$ & Thickness $t$ & Length $L$ \\
\hline $11.5 \mathrm{~mm}$ & $2 \mathrm{~mm}$ & $75 \mathrm{~mm}$ \\
\hline
\end{tabular}

Table 3: Geometry used for the validation of Equation 37

The new "non-linear" analytical solution clearly leads to a better prediction of critical stresses than the 


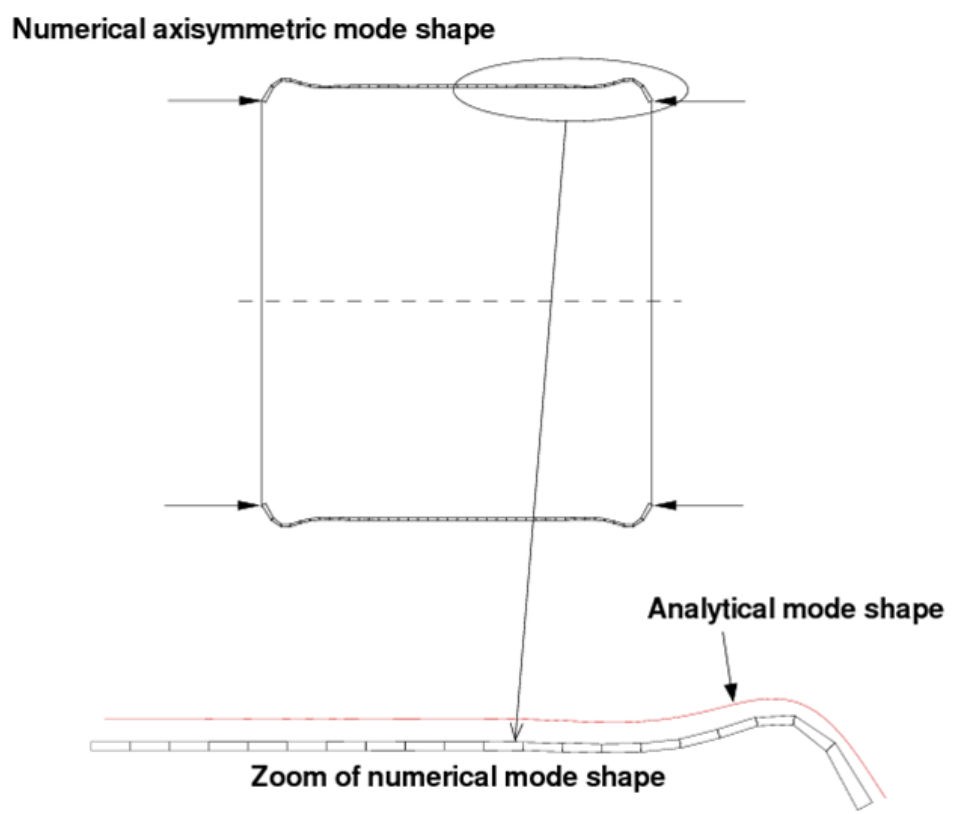

Figure 9: Comparison between analytical and numerical axisymmetric mode shapes of a cylindrical tube under axial compression with free edges

\begin{tabular}{|c|c|c|}
\hline $\begin{array}{c}\text { Young's } \\
\text { modulus } E\end{array}$ & $\begin{array}{c}\text { Poisson's } \\
\text { ratio } \nu\end{array}$ & $\begin{array}{c}\text { Isotropic } \\
\text { hardening } \\
\text { modulus } H\end{array}$ \\
\hline $70000 \mathrm{MPa}$ & 0.33 & $500-2000 \mathrm{MPa}$ \\
\hline
\end{tabular}

Table 4: Material properties used for the validation of Equation 37

classical "linearized" analytical expression, when compared to reference numerical results, with a maximum relative error of $3 \%$ instead of about 15\%. The geometric stiffness effect is thus proved to have a non negligible influence on the critical stresses, in the sense that the critical stress is actually not negligible when compared to the elastoplastic tangent modulus. Moreover, including these effects in the formulation allows one to predict the critical stresses very accurately as soon as pre-critical strains remain small.

\subsection{Finite pre-critical strains}

In practice, due to the fact that critical stresses are in the same order of magnitude than the tangent constitutive moduli, finite (plastic) strains are likely to occur before buckling, unless the yield and critical stresses are almost coincident, as was the case in the previous subsection. It is thus essential in the bifurcation analysis to distinguish between the reference and the current configuration so as to take into account the 


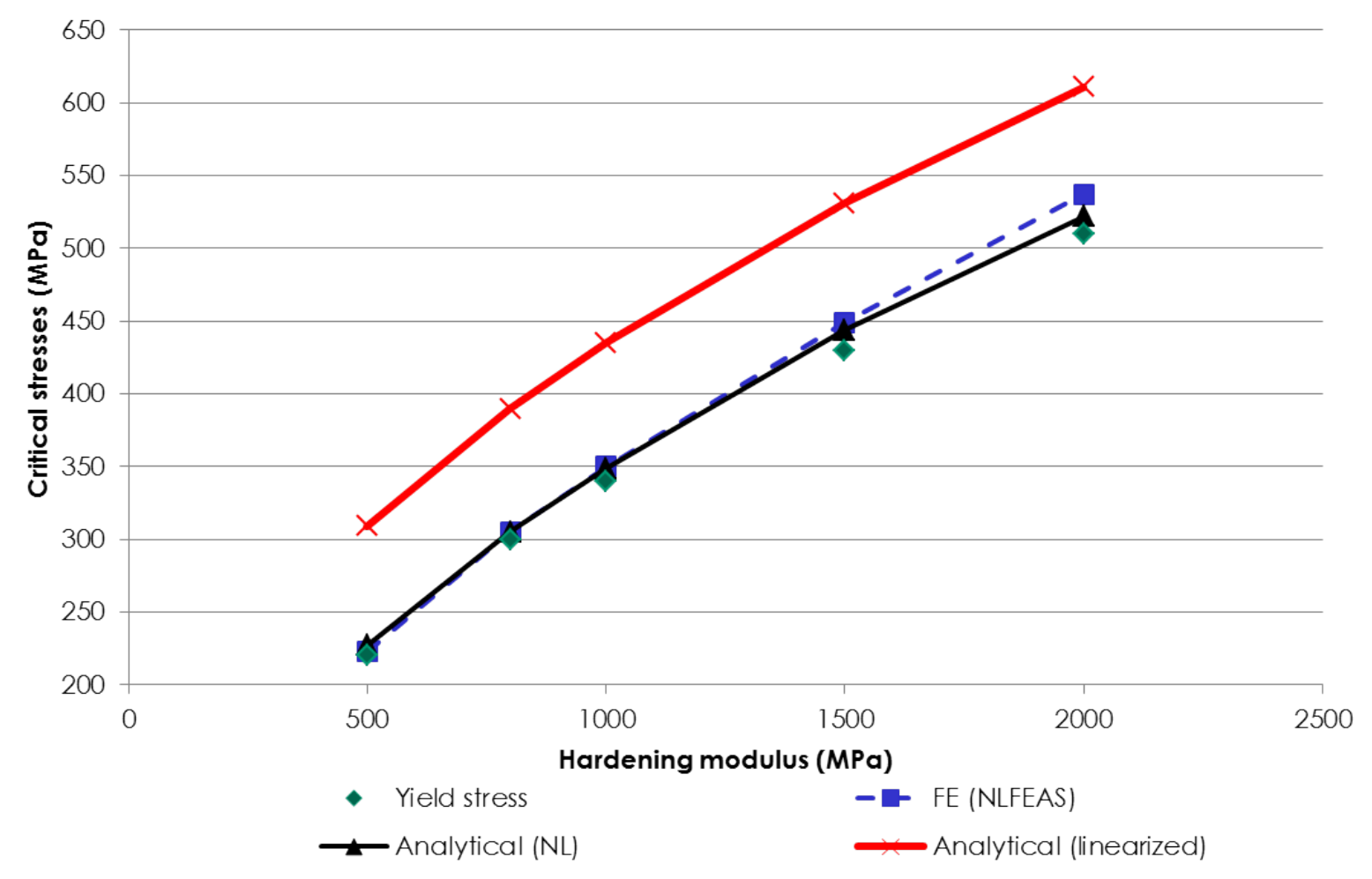

Figure 10: Comparison between analytical and numerical critical stresses: influence of the geometric stiffness

geometric changes. Ideally, in the context of finite pre-critical strains, one has to keep the complete expression for $\mathbf{K}^{p}=\mathbf{F} \cdot \mathbf{D}^{p} \cdot \mathbf{F}^{T}+(\mathbb{I} \cdot \boldsymbol{\Sigma})^{T}$ in the bifurcation equation, without replacing the deformation gradient $\mathbf{F}$ by the unit tensor, as was done previously. However, considering the exact expression for $\mathbf{F}$ would certainly lead to far too intricate governing equations for which a closed-form solution is inconceivable. Instead, in the present study, an approximate correction formula has been defined, so as to take into account the influence of finite strains, which is based on numerical observations.

For this purpose, a few computations have been performed with the same geometric and material parameters as before (see Tables 3 and 4), a constant hardening modulus $H=1000 M P a$ and various yield stresses (while verifying that the critical stress is always strictly higher than the yield stress). In all cases, it was found that the numerical second Kirchhoff critical stress was somewhat constant, whatever the yield stress considered, and equal to the "non-linear" analytical value, as seen in Figure 11. It means that the expression $\xi_{c}^{N L}$ in Equation (37) can still be used to represent fairly accurately the actual second Kirchhoff critical stress. Then, one has to find a way to derive the sought nominal critical stress (which is directly related to the critical force) from the second Kirchhoff critical stress. In finite strains, the (nominal) first Kirchhoff stress tensor is related to the second Kirchhoff stress tensor as follows:

$$
\Pi=\mathbf{F} \cdot \mathbf{\Sigma}
$$


so that the nominal critical stress may be evaluated here as:

$$
\xi_{c}^{n o m}=(1+\varepsilon) \xi_{c}^{N L}
$$

due to the uniaxial stress state.

In Equation (39), $\varepsilon$ represents the (negative) longitudinal nominal strain at critical time, which may be approximated by the Green plastic strain. In the case of a linear isotropic hardening, this current strain writes thus:

$$
\varepsilon=-\frac{\xi_{c}^{N L}-\sigma_{0}}{H}
$$

Finally, the nominal critical stress $\xi_{c}^{n o m}$, derived from Equations (37), (39) and 40 has been plotted in Figure 11 together with the second Kirchhoff critical stress $\xi_{c}^{N L}$ and their two numerical counterparts stemming from finite element computations using NLFEAS. For this purpose, a constant hardening modulus $H=1000 \mathrm{MPa}$ has been retained and various yield stresses have been considered. As mentioned before, one can observe that the numerical second Kirchhoff critical stress ("FE (PK2)") does not highly differ from the analytical prediction $\xi_{c}^{N L}$ ("Analytical (NL)"). As a consequence, the nominal critical stress $\xi_{c}^{\text {nom }}$ ("Analytical (NL+finite strains)") is found to be very close to the nominal critical stress obtained numerically ("FE (nominal)"). The procedure above seems thus to be a good way to predict analytically the critical force of a cylindrical tube under axial compression, encompassing the influence of all geometric non-linearities, including the presence of finite pre-critical strains.

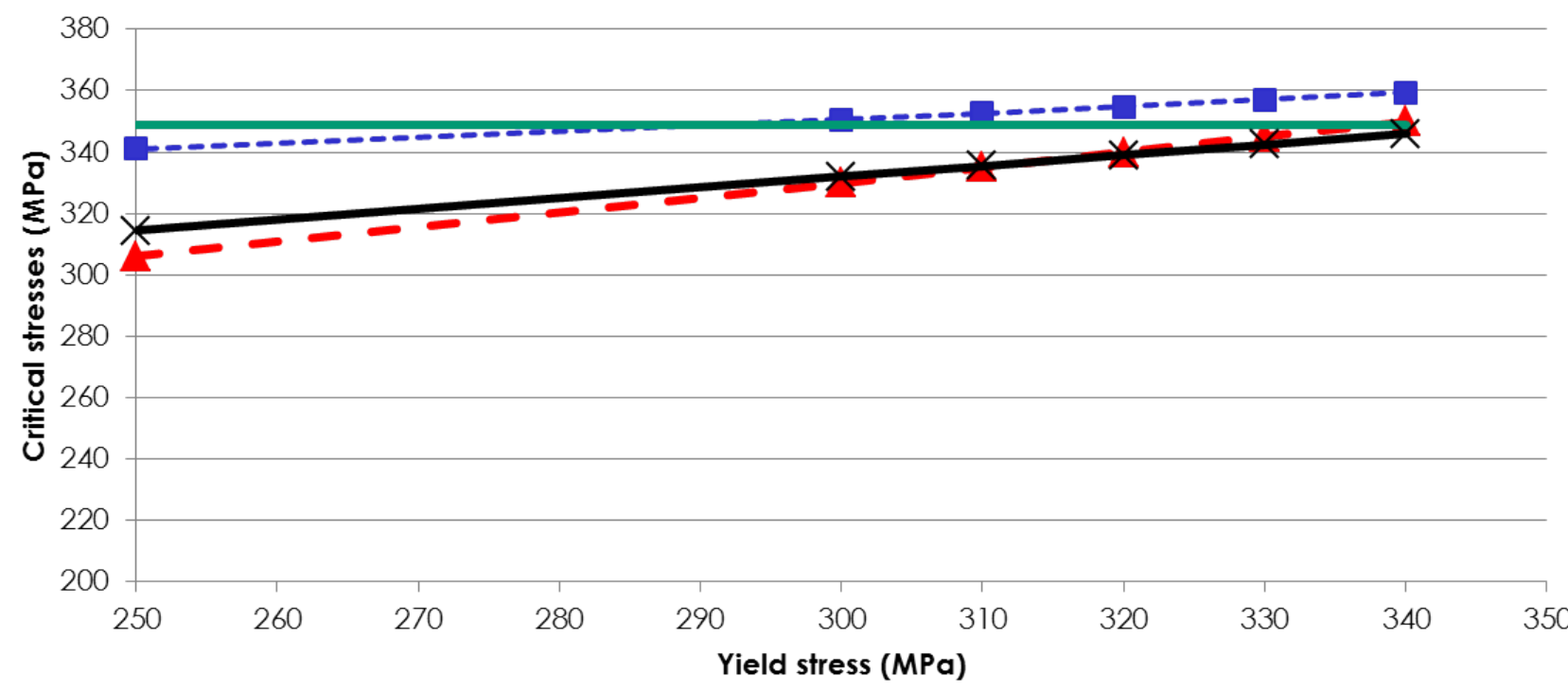

$-\Delta-\mathrm{FE}$ (nominal) $\quad-\rightarrow-F E(P K 2) \longrightarrow$ Analytical (NL) $\longrightarrow$ Analytical (NL+finite strains)

Figure 11: Comparison between analytical and numerical critical stresses: influence of the finite strains 


\subsection{Experimental/analytical validation}

The last subsection is devoted to the final comparison between the previous experimental results and the analytical approximation described above. For practical purposes, the constitutive law of the aluminum specimens, initially calibrated in terms of true stresses and strains, must be now expressed in terms of second Kirchhoff stresses and Green strains, so as to be implemented in the analytical procedure and in NLFEAS software.

The uniaxial (compression) "material" stress-strain curve is thus drawn from the experimental nominal stress-strain curve. As elastic strains remain infinitesimal, the new Young's modulus obtained through a linear regression is similar to the previous value implemented in Abaqus (see Table 1). The same standard value as before is retained for the Poisson's ratio. Regarding the plastic behavior, the new yield stress is equal to the previous value for the reasons cited above, whereas the hardening law, fitted from the material stress-(equivalent) plastic strain curve, is represented by a quite new quadratic expression. All these "new" material properties, related to the material representation of the constitutive behavior, are listed in Table 5 .

\begin{tabular}{|c|c|c|c|}
\hline $\begin{array}{c}\text { Young's } \\
\text { modulus } E\end{array}$ & $\begin{array}{c}\text { Poisson's } \\
\text { ratio } \nu\end{array}$ & $\begin{array}{c}\text { Yield } \\
\text { stress } \sigma_{0}\end{array}$ & $\begin{array}{c}\text { Hardening } \\
\text { law } \sigma(p)\end{array}$ \\
\hline $70000 M P a$ & 0.33 & $172 M P a$ & $-6100 p^{2}+2030 p+172(M P a)$ \\
\hline
\end{tabular}

Table 5: Material properties used in analytical and numerical computations (NLFEAS)

In Table 6 and Figure 12, all the previous experimental, numerical and analytical critical stresses obtained for the specimens in hand are confronted to each other. First, the critical stress computed using NLFEAS (relying on a different finite element formulation) turns out to be in very good accordance with the numerical result obtained with Abaqus and, therefore, with the experimental value. Then, three analytical predictions are examined, namely the classical analytical solution $\xi_{c}$, the modified closed-form expression $\xi_{c}^{N L}$ taking into account the geometric stiffness effects, and the final approximation also considering the influence of finite pre-critical strains. Since the hardening law is non-linear, the two expressions $\xi_{c}$ and $\xi_{c}^{N L}$, as well as $\xi_{c}^{n o m}$, involve the numerical resolution of a transcendental algebraic equation (the tangent modulus being dependent on the current stress/plastic strain). The end result is the very good accordance between the full non-linear analytical prediction with the experimental and numerical solutions (with $5 \%$ of relative error between the experimental and analytical critical stresses against $17 \%$ and $22 \%$ respectively for the two other intermediate and classical analytical values that overestimate the experimental result). Furthermore, the relative error between the full non-linear analytical solution and the numerical predictions is only $2 \%$ or even $0.4 \%$, depending on the numerical program used.

The very close agreement between all these values proves that the proposed methodology is an efficient 
analytical procedure for investigating the plastic buckling of thick cylindrical tubes under axial compression. Owing to the level of generality of the procedure implemented as for the consideration of the geometric non-linearities, it is supposed to be easily applied to other plastic buckling analyses of thick shells of various geometries with various loadings and boundary conditions, as soon as a closed-form solution is initially available in the context of linearized buckling.

\begin{tabular}{|c|c|c|c|c|c|}
\hline Experimental & $\begin{array}{c}\mathrm{FE} \\
(\text { Abaqus })\end{array}$ & $\begin{array}{c}\mathrm{FE} \\
(\mathrm{NLFEAS})\end{array}$ & $\begin{array}{c}\text { Analytical } \\
(\mathrm{NL}+\text { finite strains }) \\
\xi_{c}^{\text {nom }}\end{array}$ & $\begin{array}{c}\text { Analytical } \\
(\mathrm{NL}) \\
\xi_{c}^{N L}\end{array}$ & $\begin{array}{c}\text { Analytical } \\
(\text { linear }) \\
\xi_{c}\end{array}$ \\
\hline 267 & 274 & 280 & 281 & 312 & 327 \\
\hline
\end{tabular}

Table 6: Nominal critical stresses (in $M P a$ )

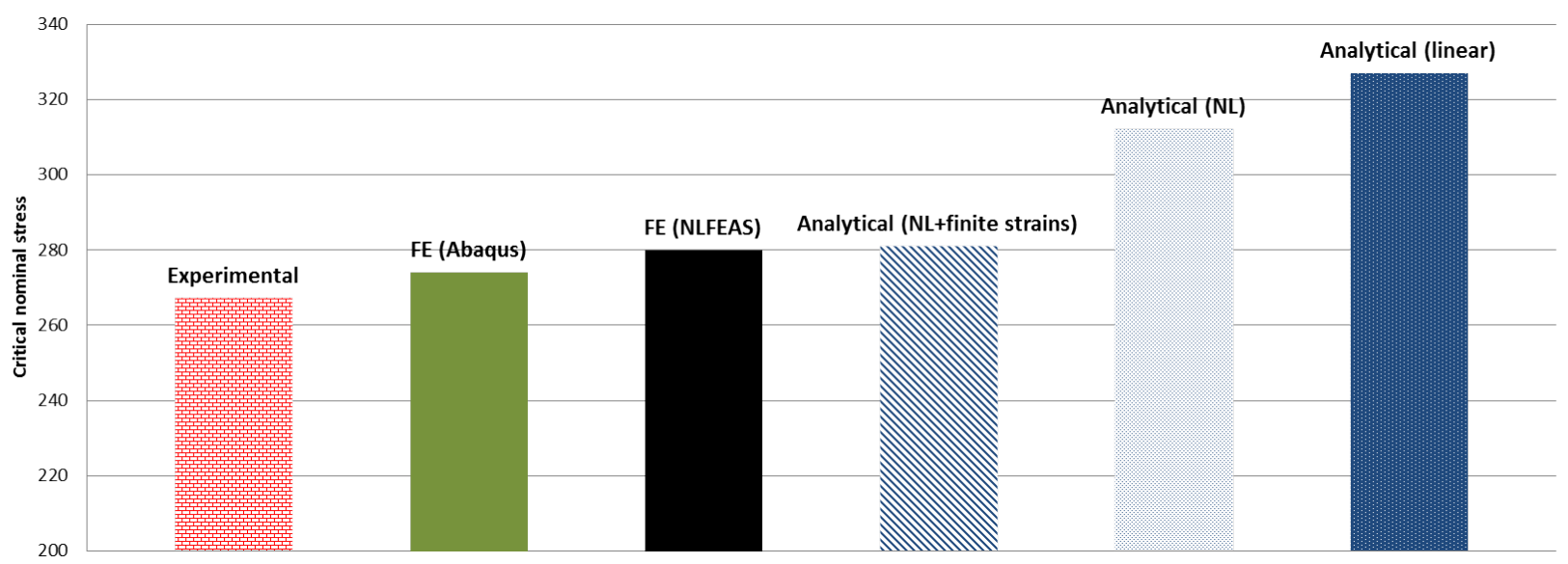

Figure 12: Comparison between analytical, numerical and experimental critical stresses

\section{Conclusion}

In this paper, a comprehensive analysis of the plastic buckling of a cylindrical shell under axial compression has been undertaken. The case of thick tubes has been particularly considered in order to minimize the effect of geometric imperfections on the critical loads. Furthermore, the choice of free edge boundary conditions allows one to remove the so-called edge effects leading to heterogeneous pre-critical stress states which may considerably reduce the critical loads encountered in practice with respect to the theoretical values obtained in idealized homogeneous pre-bifurcation conditions. Consequently, a specific experimental 
set-up has been developed and experimental compression tests have been conducted on aluminum specimens with a very low radius-to-thickness ratio.

More significantly, an analytical formulation has been proposed to investigate the elastoplastic buckling of cylindrical shells under axial compression, which allows one to take into account the effect of geometric stiffness terms in the theoretical developments in a straightforward manner, giving rise to an original closedform expression of the critical stress. Moreover, the influence of finite pre-critical strains has been included in the model in an approximate way. The validity of both consecutive solutions has been confirmed using numerical finite element computations. Lastly, the new analytical procedure has been confronted to previous experimental results, showing a very good agreement.

The proposed method is likely to apply for other shell configurations in so far as one can ignore any imperfection (as in the present case) and if one diposes of a closed-form expression for the critical load in the classical linearized framework. The present developments are devoted to be used next to analyze the influence of manufacturing process (pre-loads/pre-strains or residual stresses) on the plastic buckling response, from the three experimental, numerical and analytical points of view. This procedure should be then generalized to other configurations (cylindrical shells under external pressure, spherical shells) allowing for an efficient and accurate dimensioning of sub-marine structures, among others.

\section{Acknowledgements}

The authors are indebted to the DGA (Direction Générale de l'Armement) for its financial support.

\section{References}

[1] Hill, R., A general theory of uniqueness and stability in elastic-plastic solids, Journal of Mechanics and Physics of Solids 6 (1958) 236-249.

[2] Cimetière, A., Sur la modélisation et le flambage des plaques élastoplastiques (On the modelling and buckling of elastoplastic plates), PhD Thesis (University of Poitiers, France, 1987).

[3] Shanley, F.R., Inelastic column theory, International Journal of Aeronautical Sciences 14 (1947) 261-267.

[4] Hao, P., Wang, B., Tian, K., Li, G., Sun, Y. and Zhou, C., Fast procedure for non-uniform optimum design of stiffened shells under buckling constraint, Structural and Multidisciplinary Optimization 55 Issue 4 (2017) 1503-1516.

[5] Bushnell, D., Plastic buckling, Pressure vessels and piping: design technology - A decade of progress (S.Y. Zamrik and D. Dietrich, 1982) Book No. G00213 47-117.

[6] Bushnell, D., Computerized buckling analysis of shells (M. Nijhoff, 1985).

[7] Hutchinson, J.W., Plastic buckling, Advances in Applied Mechanics 14 (1974) 67-144.

[8] Gellin, S., Effect of an axisymmetric imperfection on the plastic buckling of an axially compressed cylindrical shell, Journal of Applied Mechanics 46 (1979) 125-131.

[9] Kyriakides, S., Bardi, F.C. and Paquette, J.A., Wrinkling of circular tubes under axial compression: Effect of anisotropy, Journal of Applied Mechanics 72 (2005) 301-305. 
[10] Bardi, F.C. and Kyriakides, S., Plastic buckling of circular tubes under axial compression - part I: Experiments, International Journal of Mechanical Sciences 48 (2006) 830-841.

[11] Bardi, F.C., Kyriakides, S. and Yun, H.D., Plastic buckling of circular tubes under axial compression - part II: Analysis, International Journal of Mechanical Sciences 48 (2006) 842-854.

[12] Ore, E. and Durban, D., Elastoplastic buckling of axially compressed circular cylindrical shells, International Journal of Mechanical Sciences 34 Issue 9 (1992) 727-742.

[13] Tvergaard, V., On the transition from a diamond mode to an axisymmetric mode of collapse in cylindrical shells, International Journal of Solids and Structures 19 Issue 10 (1983) 845-856.

[14] Tvergaard, V., Plastic buckling of axially compressed circular cylindrical shells, Thin-Walled Structures 1 Issue 2 (1983) $139-163$

[15] Christoffersen, J. and Hutchinson, J.W., A class of phenomenological corner theories of plasticity, Journal of the Mechanics and Physics of Solids 27 Issues 5-6 (1979) 465-487.

[16] Batterman, S.C., Plastic buckling of axially compressed cylindrical shells, AIAA Journal 3 Issue 2 (1965) $316-325$.

[17] Batterman, S.C., Free-edge plastic buckling of axially compressed cylindrical shells, Journal of Applied Mechanics 35 (1968) $73-79$.

[18] Mao, R. and Lu, G., Plastic buckling of circular cylindrical shells under combined in-plane loads, International Journal of Solids and Structures 38 (2001) 741-757.

[19] Peek, R., Axisymmetric wrinkling of cylinders with finite strain, Journal of Engineering Mechanics 126 Issue 5 (2000) $455-461$.

[20] Wang, B., Zhu, S., Hao, P., Bi, X., Du, K., Chen, B., Ma, X. and Chao, Y.J., Buckling of quasi-perfect cylindrical shell under axial compression: A combined experimental and numerical investigation, International Journal of Solids and Structures 130-131 (2018) 232-247.

[21] Stein, M., The effect on the buckling of perfect cylinders of prebuckling deformations and stresses induced by edge support, Collected Papers on Instability of Shell Structures (NASA TN D-1510, 1962).

[22] Almroth, B.O., Influence of edge conditions on the stability of axially compressed cylindrical shells, AIAA Journal 4 Issue 1 (1966) 134-140.

[23] Alhussainy, F., Sheikh, M.N. and Hadi, M.N.S., Behaviour of small diameter steel tubes under axial compression, Structures 11 (2017) 155-163.

[24] Le Grognec, P. and Le van, A., Some new analytical results for plastic buckling and initial post-buckling of plates and cylinders under uniform compression, Thin-Walled Structures 47 Issues 8-9 (2009) 879-889.

[25] Halphen, B. and Nguyen, Q.S., Sur les matériaux standard généralisés (On the generalized standard materials), Journal de Mécanique 14 Issue 1 (1975) 39-63.

[26] Germain, P., Nguyen, Q.S. and Suquet, P., Continuum thermodynamics, Journal of Applied Mechanics 50 (1983) 10101020 .

[27] Green, A.E. and Naghdi, P.M., A general theory of an elastic-plastic continuum, Archive for Rational Mechanics and Analysis 18 (1965) 251-281.

[28] Green, A.E. and Naghdi, P.M., Some remarks on elastic-plastic deformation at finite strain, International Journal of Engineering Sciences 9 (1971) 1219-1229.

[29] Hutchinson, J.W., On the post-buckling behavior of imperfection-sensitive structures in the plastic range, Journal of Applied Mechanics (1972) 155-162.

[30] Nguyen, Q.S., Stability and Non-Linear Solid Mechanics (Wiley, 2000).

[31] Donnell, L.H., Beams, plates and shells (Mc Graw-Hill, 1976).

[32] Timoshenko, S.P. and Gere, J.M., Theory of elastic stability (Mc Graw-Hill, 1961). 
[33] Le Grognec, P. and Le van, A., Elastoplastic bifurcation and collapse of axially loaded cylindrical shells, International Journal of Solids and Structures 45 Issue 1 (2008) 64-86. 